\title{
BEAM OPERATION WITH CRAB CAVITIES AT KEKB
}

\author{
T. Abe, T. Agho, K. Akai, M. Akemoto, A. Akiyama, M. Arinaga, K. Ebihara, K. Egawa,
}

A. Enomoto, J. Flanagan, S. Fukuda, H. Fukuma, Y. Funakoshi, K. Furukawa, T. Furuya, K. Hara,

T. Higo, S. Hiramatsu, H. Hisamatsu, H. Honma, T. Honma, K. Hosoyama, T. Ieiri, N. Iida,

H. Ikeda, M. Ikeda, S. Inagaki, S. Isagawa, H. Ishii, A. Kabe, E. Kadokura, T. Kageyama,

K. Kakihara, E. Kako, S. Kamada, T. Kamitani, K. Kanazawa, H. Katagiri, S. Kato, T. Kawamoto,

S. Kazakov, M. Kikuchi, E. Kikutani, K. Kitagawa, H. Koiso, Y. Kojima, I. Komada, T. Kubo,

K. Kudo, N. Kudo, K. Marutsuka, M. Masuzawa, S. Matsumoto, T. Matsumoto, S. Michizono,

K. Mikawa, T. Mimashi, S. Mitsunobu, K. Mori, A. Morita, Y. Morita, H. Nakai, H. Nakajima,

T. T. Nakamura, H. Nakanishi, K. Nakanishi, K. Nakao, S. Ninomiya, Y. Ogawa, K. Ohmi,

S. Ohsawa, Y. Ohsawa, Y. Ohnishi, N. Ohuchi, K. Oide, M. Ono, T. Ozaki, K. Saito, H. Sakai,

Y. Sakamoto, M. Sato, M. Satoh, K. Shibata, T. Shidara, M. Shirai, A. Shirakawa, T. Sueno,

M. Suetake, Y. Suetsugu, R. Sugahara, T. Sugimura, T. Suwada, O. Tajima, S. Takano, S. Takasaki,

T. Takenaka, Y. Takeuchi, M. Tawada, M. Tejima, M. Tobiyama, N. Tokuda, S. Uehara, S. Uno,

Y. Yamamoto, Y. Yano, K. Yokoyama, Ma. Yoshida, Mi. Yoshida, S. Yoshimoto, K. Yoshino, KEK, Oho, Tsukuba, Ibaraki 305-0801, Japan

E. Perevedentsev, D. N. Shatilov, BINP, Novosibirsk, Russia

\section{Abstract}

KEKB has been operating with crab cavities for the first time in the world. This paper represents beam operation with the crab crossing.

\section{INTRODUCTION}

The KEKB B-factory is an energy-asymmetric doublering collider with the center-of-mass energy at $\Upsilon(4 S)$ [1]. An 8-GeV electron ring (HER) and a $3.5-\mathrm{GeV}$ positron ring (LER) have a single interaction point (IP) where beams collide with a horizontal crossing angle of $22 \mathrm{mrad}$. No serious problems due to the crossing angle have been observed in the range of the vertical beam-beam tune shift parameter $\xi_{y} \sim 0.05$. However, the recent beam-beam simulation has suggested that head-on collision will improve $\xi_{y}$ significantly $\left(\xi_{y}>0.1\right)$ [2]. In order to recover an effective headon collision at KEKB, crab cavities have been installed in both rings (one cavity per ring) and beam operation has been on the way since February 2007. An overview and main subjects of the crab crossing at KEKB are presented elsewhere in these proceedings[3]. This paper reports details on beam tuning with crab cavities.

\section{COLLISION TUNING}

\section{Currents and Bunch Spacing}

The collision tuning was done in the following three modes:

- Mode-I) Investigate beam-beam performance with high bunch currents (up to $1.5 \mathrm{~mA}$ in the LER), a small number of bunches (50 or 100 bunches), and long bunch spacing ( $98 \mathrm{nsec}=49 \mathrm{rf}$ buckets). The to-

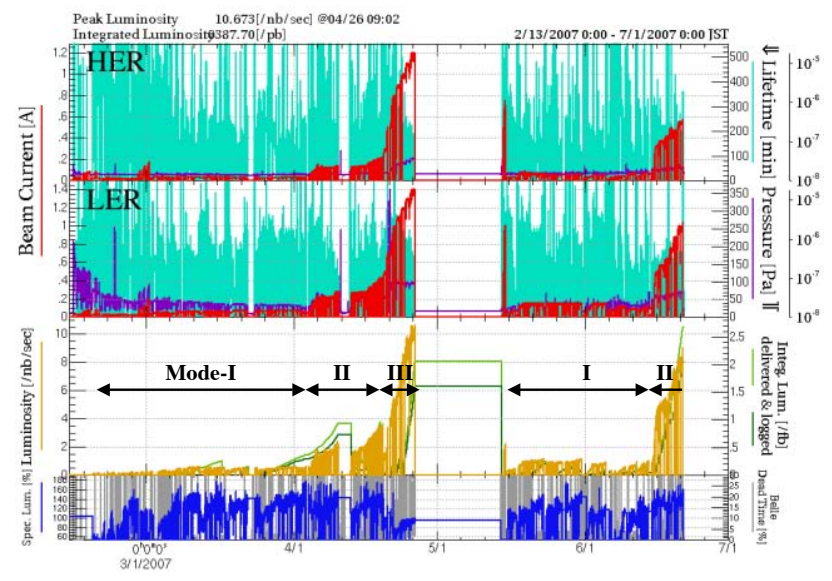

Figure 1: Operation history of KEKB with crab cavities. The rows are the stored current, the beam lifetime and the average pressure in the HER, those in the LER, the luminosity, and the specific luminosity ratio, top to bottom.

tal beam currents were limited to avoid any troubles on the crab cavities.

- Mode-II) Increase the total currents to check hardware performance of the crab cavities. The number of bunches was gradually increased, keeping bunch current as high as in Mode-I.

- Mode-III) Increase currents as high as before with the crab cavities detuned. If it is possible, the operation mode can be freely switched on and off the crab crossing for collision study and physics run.

So far most of the machine time has been spared for Mode-I as shown in Fig. 1. 


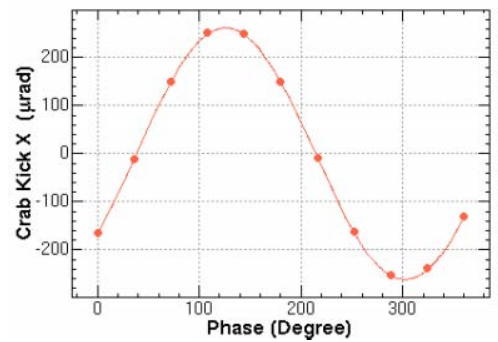

Figure 2: Dependence of the horizontal kick by the crab cavity on the rf phase in the LER. Calibration of the $\mathrm{rf}$ phase is not yet done in this figure.

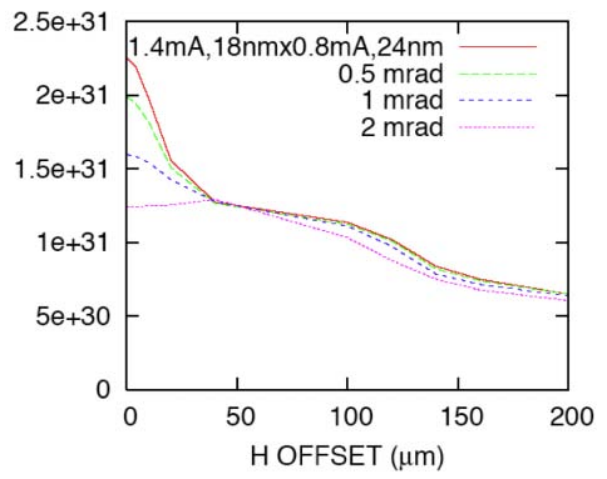

Figure 3: Simulated dependence of the luminosity on the half crossing angle and the horizontal offset. According to these simulations, the horizontal offset must be less than 20 $\mu \mathrm{m}$ to see the luminosity enhancement by the crab crossing, while the horizontal beam size at the IP is estimated to be $\sim 100 \mu \mathrm{m}$.

\section{Crab Kick}

The horizontal kick by the crab cavity was directly measured by the orbit distortion with $\sim 450$ beam position monitors all over the ring, changing the rf phase of the crab cavity as shown in Fig. 2. The kick angles estimated by the orbit response agree well with those from the setting voltage of the crab cavity, typically within $4 \%$.

\section{Crossing Angle and Horizontal Offset}

As shown in Fig. 3, the luminosity should be significantly degraded by small errors in the crab crossing mode. Therefore it is important to keep both the crossing angle and the horizontal offset between two beams sufficiently small. The crossing angle was optimized by adjusting the crab kick voltage. The horizontal offset was maintained by an orbit feedback to keep the canonical beam-beam kick constant[1]. The target position was determined by the offset scan as shown in Fig. 4

\section{Beta Function}

In most cases, the beta functions at the IP were set to be same in both rings $\left(\beta_{x}^{*} / \beta_{y}^{*}=0.8 / 0.0059 \mathrm{~m}\right)$. A higher $\beta_{x}^{*}$ than before the crab was selected to decrease the crab volt-

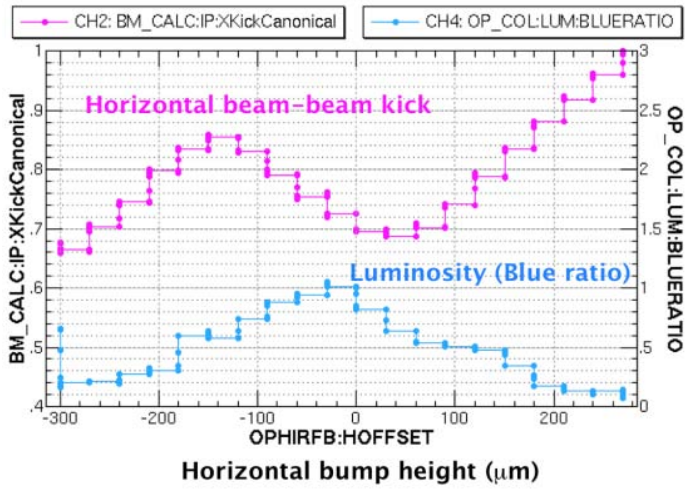

Figure 4: Scan of the horizontal offset to find the head-on condition, which locates at the offset $\approx-80 \mu \mathrm{m}$ in this case.

age. The horizontal beta function at the crab cavity $\beta_{x, \mathrm{crab}}$ was adjusted to meet the crab voltage (typically $85 / 162$ $\mathrm{m}$ for $.88 / 1.45 \mathrm{MV}$ in LER/HER). The adjustment of $\beta_{x}^{*}$ and $\beta_{x, \text { crab }}$ can be done locally and independently in the Tsukuba or Nikko straight section, respectively.

\section{Emittance and Momentum Compaction Factor}

Various settings of the horizontal emittance $\varepsilon_{x}$ and the momentum compaction factor $\alpha$ listed in Table 1 have been tried to obtain higher luminosity. The optics with negative$\alpha$ were tested to weaken the effect of the synchrotronbetatron resonance and also to suppress the bunch lengthening. In those optics, the resonances were not $2 \nu_{x}+\nu_{s}=$ integer, but $2 \nu_{x}-\nu_{s}=$ integer. The negative- $\alpha$ optics in the HER made possible to move the horizontal tune closer to the half integer. However, in the LER, the negative- $\alpha$ optics induced longitudinal oscillation due to the single bunch microwave instability. Then the negative- $\alpha$ optics were abandoned at this time. This oscillation was not observed or smaller in the negative- $\alpha$ operation in 2003.

\section{Specific Luminosity}

The specific luminosity for various optics are shown in Fig. 5. Higher horizontal emittance was tried since the bunch currents were limited by shortening of the beam lifetime at collision. In order to suppress the beam size blowup due to the synchrotron-betatron resonance and to move the horizontal tune closer to the half integer, better settings of setxupoles are inevitable[4]. An effective method for sextupole optimization has been developed[5].

The crab crossing has improved the vertical beam-beam parameter up to $\sim 0.088$, compared with 0.052 in collision with the crossing angle. Typical beam parameters in the crab crossing are listed in Table 2. The set (1) is same as the standard setting without crab crossing. For further improvement of the luminosity, it may be necessary to correct machine errors more precisely such as residual $\mathrm{x}-\mathrm{y}$ coupling. Also more intelligent tuning methods should be devised among many tuning knobs and a small number of observables[3]. 
Table 1: Optical parameters tested for the crab crossing.

\begin{tabular}{||l|c|c|c|c|c|c|c|c|c|c||}
\hline \hline & $(1)$ & $(2)$ & $(3)$ & $(4)$ & $(5)$ & $(6)$ & $(7)$ & $(8)$ & $(9)$ & \\
\hline$\varepsilon_{x}(\mathrm{HER})$ & 24 & 24 & 24 & 24 & 29 & 34 & 24 & 24 & 24 & $\mathrm{~nm}$ \\
$\varepsilon_{x}(\mathrm{LER})$ & 18 & 24 & 24 & 24 & 24 & 18 & 24 & 24 & 24 & $\mathrm{~nm}$ \\
$\alpha(\mathrm{HER})$ & 3.4 & 3.4 & 4.4 & 1.3 & 3.0 & 1.6 & -3.4 & -3.4 & -3.4 & $\times 10^{-4}$ \\
$\alpha(\mathrm{LER})$ & 3.3 & 3.4 & 3.4 & 3.4 & 3.4 & 3.3 & 3.4 & -3.4 & -2.6 & $\times 10^{-4}$ \\
$\nu_{s}(\mathrm{HER})$ & -.0226 & -.0224 & -.0264 & -.0113 & -.0215 & -.0125 & .0226 & .0226 & .0226 & \\
$\nu_{s}(\mathrm{LER})$ & -.0246 & -.0250 & -.0250 & -.0250 & -.0250 & -.0246 & -.0250 & .0249 & .0215 & \\
$V_{c, \text { acc }}(\mathrm{HER})$ & 15 & 15 & 15 & 10 & 15 & 10 & 15 & 15 & 15 & $\mathrm{MV}$ \\
$V_{c, \text { acc }}(\mathrm{LER})$ & 8 & 8 & 8 & 8 & 8 & 8 & 8 & 8 & 8 & $\mathrm{MV}$ \\
\hline
\end{tabular}

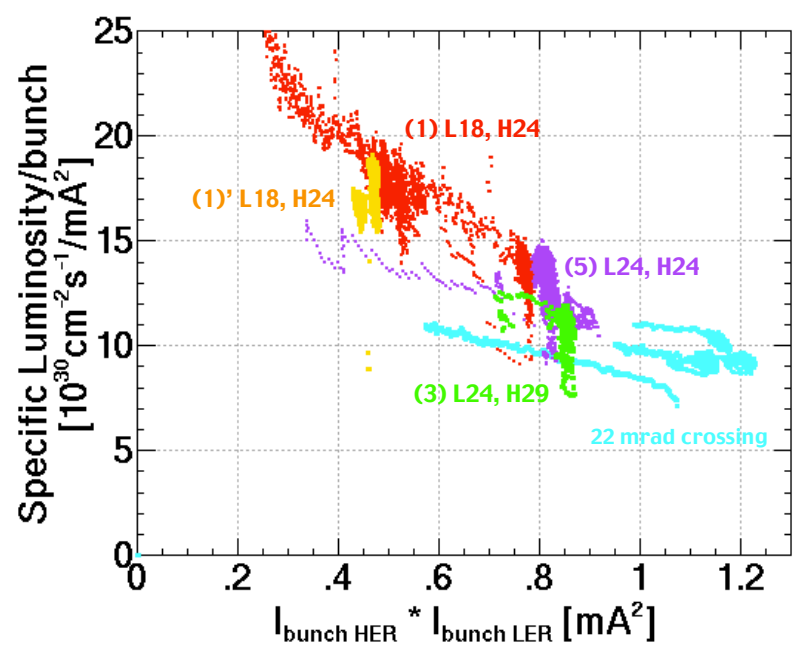

Figure 5: Specific luminosity. The horizontal emittances are: (1) and (1)' $18 / 24$, (2) $24 / 24$, (5) $24 / 29 \mathrm{~nm}$ in the LER/HER. Other parameters are listed in Table 1.

Table 2: Typical parameters for the crab crossing. As predicted by the simulation, higher vertical tunes brought about higher luminosity.

\begin{tabular}{||l|c|c|c||}
\hline \hline & LER & HER & \\
\hline$\theta_{x}$ & \multicolumn{2}{|c||}{22} & $\mathrm{mrad}$ \\
$\beta_{x}^{*}$ & 80 & 84 & $\mathrm{~cm}$ \\
$\beta_{y}^{*}$ & 0.59 & 0.65 & $\mathrm{~cm}$ \\
$\sigma_{z}$ & $\sim 8$ & $\sim 6$ & $\mathrm{~mm}$ \\
$\beta_{x, \mathrm{crab}}$ & 73 & 162 & $\mathrm{~m}$ \\
$\mu_{x} / 2 \pi$ & 0.505 & 0.511 & \\
$\mu_{y} / 2 \pi$ & 0.590 & 0.590 & \\
$\psi_{x, \mathrm{crab}} / 2 \pi$ & $\sim 0.25$ & $\sim 0.25$ & \\
$V_{c, \mathrm{crab}}$ & 0.95 & 1.45 & $\mathrm{MV}$ \\
$\omega_{\mathrm{rf}} / 2 \pi$ & \multicolumn{2}{|c|}{509} & $\mathrm{MHz}$ \\
Current & 60.9 & 33.3 & $\mathrm{~mA}$ \\
Bunches & \multicolumn{2}{|c|}{50} & \\
$\sigma_{x}^{*}$ & 120 & 140 & $\mu \mathrm{m}$ \\
$\sigma_{y}^{*}$ & 1.3 & 1.3 & $\mu \mathrm{m}$ \\
$\xi_{x}$ & 0.115 & 0.111 & \\
$\xi_{y}$ & 0.108 & 0.088 & $10^{32} \mathrm{~cm}^{-2} \mathrm{~s}^{-1}$ \\
Luminosity & \multicolumn{2}{|c|}{5.9} \\
\hline \hline
\end{tabular}

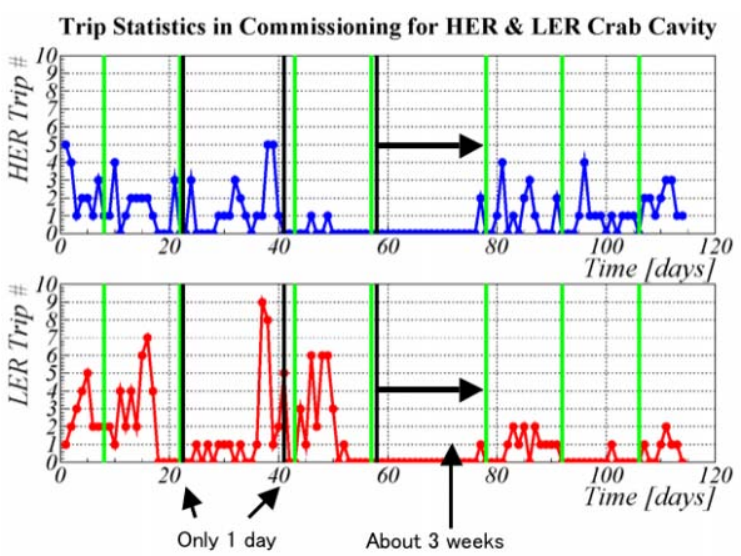

Figure 6: History of the number of trips per day of the crab cavities. The green and black lines show the maintenance and the warmup of the cavities, respectively.

\section{HIGH CURRENT OPERATION}

The crab crossing with higher beam currents has been in progress in Mode-II since mid-June. More than $1 \mathrm{~A}$ in 1389 bunches with $3.5 \mathrm{rf}$-bucket spacing, which is the standard fill pattern for the physics run, has been stored in the LER. So far no serious troubles have occurred on the crab cavities. The trip rates are sufficiently low in both rings even with high currents as shown in Fig. 6. The beam currents plan to be increased to the nominal values for the physics run with the crab cavities detuned.

\section{ACKNOWLEDGMENT}

The authors thank all people who have supported the commissioning of the crab crossing at KEKB.

\section{REFERENCES}

[1] KEKB B-Factory Design Report., KEK Report 95-7,1995. Nucl, Instrum, Meth. A499, 2003.

[2] K. Ohmi et. al., Phys. Rev. Lett. 92, 21401(2004).

[3] K. Oide et. al., in these proceedings.

[4] Y. Funakoshi et. al., in these proceedings.

[5] A. Morita et. al., in these proceedings. 